\title{
Towards the phase A review of MAORY, the multi-conjugate adaptive optics module for the E-ELT
}

Emiliano Diolaiti ${ }^{1, a}$, Jean-Marc Conan ${ }^{2}$, Italo Foppiani ${ }^{3}$, Matteo Lombini ${ }^{1,3}$, Enrico Marchetti ${ }^{4}$, Cyril Petit $^{2}$, Clélia Robert ${ }^{2}$, Laura Schreiber ${ }^{3}$, Andrea Baruffolo ${ }^{5}$, Michele Bellazzini ${ }^{1}$, Giovanni Bregoli ${ }^{1}$, Paolo Ciliegi ${ }^{1}$, Giuseppe Cosentino ${ }^{3}$, Valdemaro Biliotti ${ }^{6}$, Sandro D'Odorico ${ }^{4}$, Thierry Fusco ${ }^{2}$, Norbert Hubin $^{4}$, Serge Meimon ${ }^{2}$, and Jean-François Sauvage ${ }^{2}$

1 INAF - Osservatorio Astronomico di Bologna, via Ranzani 1, 40127 Bologna, Italy

2 Office National d'Etudes et de Recherches Aerospatiales, B.P. 72, 92322 Châtillon cedex, France

3 Università di Bologna - Dipartimento di Astronomia, via Ranzani 1, 40127 Bologna, Italy

4 European Southern Observatory, K. Schwarzschild Str. 2, 85748 Garching b. Muenchen, Germany

5 INAF - Osservatorio Astronomico di Padova, vicolo dell'Osservatorio 5, 35122 Padova, Italy

6 INAF - Osservatorio Astrofisico di Arcetri, largo E. Fermi 5, 50125 Firenze, Italy

\begin{abstract}
MAORY is the multi-conjugate adaptive optics module for the European Extremely Large Telescope. A conceptual design study is in progress and is approaching the final review. A description of the current design and of the expected performance is presented.
\end{abstract}

\section{Introduction}

MAORY (acronym for Multi-conjugate Adaptive Optics RelaY) is the Multi-Conjugate Adaptive Optics (MCAO) module for the European Extremely Large Telescope (E-ELT, Gilmozzi \& Spyromilio [1]). A two years phase A study is in progress, in the framework of the E-ELT instrumentation studies (Hubin [2]) sponsored by the European Southern Observatory (ESO). The study is performed by a consortium including the Italian National Institute for Astrophysics (INAF) and the Office National d'Etudes et de Recherches Aerospatiales (ONERA); ESO contributes to specific aspects of the study, concerning in particular key hardware components such as detectors and deformable mirrors.

MAORY has to provide a corrected Field of View (FoV) of medium to large size (up to 2 arcmin), over the baseline wavelength range $0.8-2.4 \mu \mathrm{m}$. It is expected to achieve a correction of high quality and uniformity with high sky coverage. With a seeing of 0.8 arcsec in the visible, the Strehl Ratio (SR) at $\lambda=2.16 \mu \mathrm{m}$, averaged across the field of view, is expected to be approximately 0.5 over $50 \%$ of the sky at the Galactic Pole.

In order to achieve the high performance required, the wavefront sensing system is based on Laser Guide Stars (LGS), following the same approach used in other systems for present and future telescopes (Rigaut et al. [3], Ellerbroek [4], Herriot et al. [5]). The wavefront correction is performed by two post-focal Deformable Mirrors (DMs), that complement the ground-layer and the tip-tilt correction provided by the E-ELT adaptive mirrors M4 and M5.

The current design and the expected performance of MAORY are presented in this paper, while the on-going Phase A study is approaching its final review. Since the study is not completed at the moment of this writing, the concept and the results presented here are not fully consolidated yet.

\section{Design overview}

MAORY is designed to be located on the E-ELT Nasmyth platform, on one of the bent foci. An overall view of the module is shown in figure 1.

\footnotetext{
a e-mail: emiliano.diolaiti@oabo.inaf.it
} the original work is properly cited. 


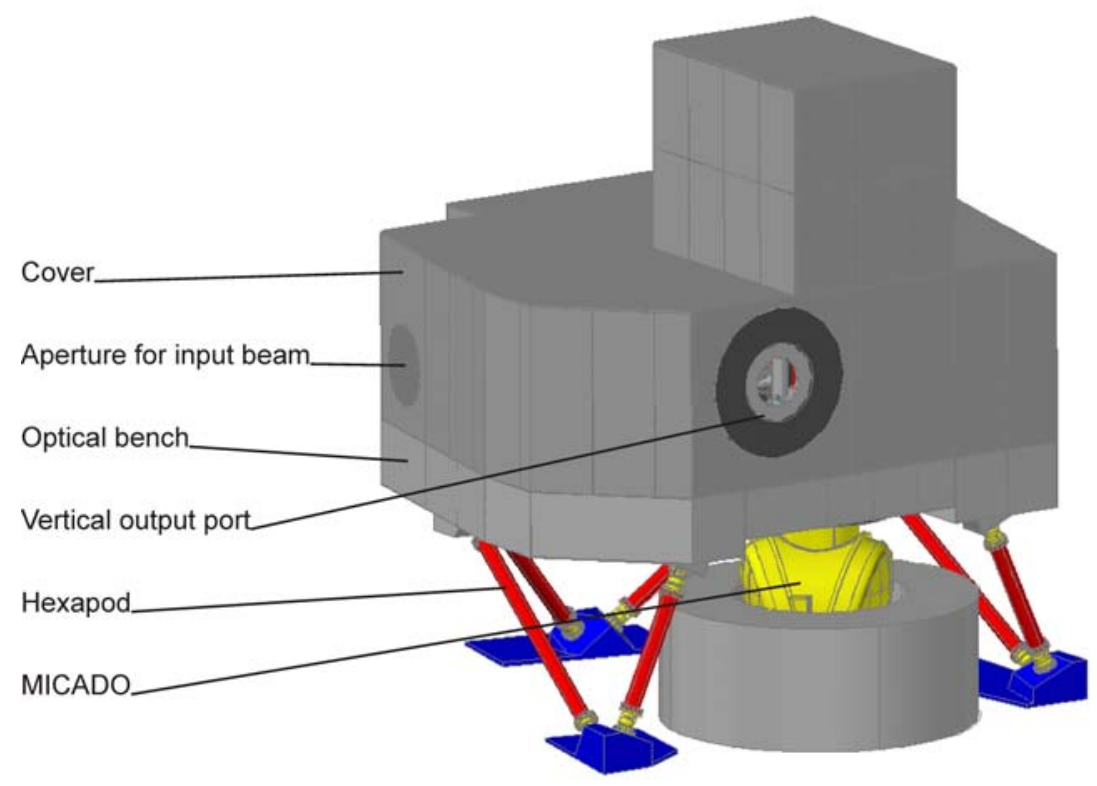

Fig. 1. Overall view of MAORY. The optical bench is supported by a hexapod. Underneath the bench, the cryostat of the client instrument MICADO is shown; the hollow cylinder around it represents the rotational volume of the electronics cabinets of MICADO. The hole in the cover in the foreground is the so-called vertical port, for a detached instrument on the Nasmyth platform.

From the optical design point of view, the MCAO module is a finite conjugate relay formed by two pairs of aspheric off-axis mirror. Three flat mirrors (two of which deformable) allow to fold the relay and to fit the reserved area on the Nasmyth platform. The optical relay makes an image of the telescope focal plane with unit magnification.

The module feeds two focal stations: a gravity invariant port (or 'horizontal'port) underneath the optical bench, providing mechanical derotation for a light instrument $(<5$ tons $)$, and a vertical port on one side of the bench to feed an instrument standing on the Nasmyth platform, detached from the module. The foreseen client instrument on the gravity invariant port is MICADO, a high-angular resolution camera (Davies et al. [6]).

A thermal analysis has been performed to evaluate the module emissivity. It has been found that, assuming a high performance coating as that recalled in Foppiani et al. [7], the MCAO module thermal background is smaller than $50 \%$ of the global background contribution due to sky and telescope together at any ambient temperature $T<24^{\circ} \mathrm{C}$. On the basis of this analysis, the baseline is not to cool the MCAO module.

A more detailed description of the post-focal relay optical and mechanical design is presented in Foppiani et al. [7].

Three levels of wavefront correction are implemented: the telescope adaptive mirror M4, conjugated to few hundred meters above the telescope pupil, and two post-focal DMs, conjugated at different ranges in the atmosphere ( $4 \mathrm{~km}$ and $12.7 \mathrm{~km}$ ). The tip-tilt correction is mainly performed by the telescope mirror M5.

High-order wavefront sensing is performed through 6 Sodium LGSs, arranged on a circle of 2 arcmin diameter. This angular separation has been found to be a good compromise between errors related to the LGS cone effect (that push towards larger launching angles) and isoplanatic effects (Conan et al. [8]). The LGSs are projected from the telescope edge; this choice translates into a slightly higher measurement error than for central projection, due to the larger spot elongation, however it allows to get rid of the so-called fratricide effect among different LGSs, related to the Rayleigh scattering in 
the atmosphere. The splitting of the science and LGS beams in the optical relay is accomplished by means of a dichroic, that transmits the LGS light. The LGS beam is focused by a refractive objective, that creates a F/5.1 focus, reducing the travel for refocusing when the Zenith distance and the Sodium range change. The 6 LGS feed the LGS WaveFront Sensor (LGS WFS), that is based on the Shack-Hartmann scheme combined with Continuous Wave lasers. Other wavefront sensing schemes are under investigation, to mitigate the quite demanding requirements in terms of laser power and detector size imposed by the baseline approach.

To solve the LGS tip-tilt indetermination problem, also 3 Natural Guide Stars (NGS) are used (Ellerbroek \& Rigaut [9]): as a baseline two of them are used to measure tip-tilt only, while the third, positioned on the brightest star found on the search FoV, is used to measure tip-tilt and focus, in order to provide a reference for the rapidly variable focus term in the LGS signals, due to the Sodium layer instability. The $3 \mathrm{NGS}$ are searched on a wide FoV 2.6 arcmin and are observed in the near IR ( $\mathrm{H}$ band), in order to take advantage of the spot shrinking ensured by the high-order correction driven by the LGS WFS, allowing the use of faint NGS that translate into a high sky coverage. An additional complication on the NGS WFS design is the need to measure the tip-tilt due to the telescope windshake, characterized by a large amplitude; a Kalman filter is planned in order to take advantage of the relatively high degree of temporal correlation of this phenomenon. On each of the 3 NGS WFS channels, the light of wavelength $0.6-0.9 \mu \mathrm{m}$ is sent to a so-called Reference WFS (RWFS), that operates typically at frequencies in the range $0.1-1 \mathrm{~Hz}$, used to monitor the LGS non common path aberrations related to the Sodium layer profile variability (Pfrommer et al. [10]). During normal operations the RWFS has a pupil sampling of $\sim 10 \times 10$ subapertures. In an engineering mode the RWFS may work with up to $84 \times 84$ subapertures, in order to perform a MCAO correction based on 3 NGS.

The LGS constellation is kept fixed with respect to the telescope pupil, so that it rotates with the elevation axis as seen from the Nasmyth platform. The post-focal DMs are rotated at the same rate. In this way the LGS WFS and all DMs (post-focal and telescope M4) are reciprocally fixed. This property allows to relax the requirement on the update rate of the control matrix in the real-time computer, since the only residual variation is the diameter of the LGS footprints, projected on the high-altitude layers, that implies a control matrix update rate in the order of 140s. An alternative strategy is to avoid rotating the post-focal DMs; the wavefront correction is computed on 'virtual'DMs and then rotated onto the actual ones. This strategy, although it simplifies the opto-mechanics, has to be further investigated, in particular concerning the calibration aspects. In any case the NGS WFS measurements have to be derotated in order to take into account the reciprocal rotation between the NGS WFS and the telescope M4/M5; considering that the NGS WFS measurements are concerned with the lowest orders of the wavefront distortions, this aspect is not considered critical.

\section{Expected performance}

The MCAO performance has been evaluated by means of a Power Spectral Density (PSD) based analytic code, complemented by a correction factor to account for the unseen turbulence due to the LGS cone effect. Details about the modeling tool may be found in Petit et al. [11]. This tool has been used to estimate the residual phase PSD after MCAO correction over a grid of positions across the FoV; from the PSDs, the Point Spread Functions (PSF) at different wavelengths have been evaluated. A sample PSF is shown in figure 2.

The PSFs have been used to derive performance statistics across different field diameters and for different wavelengths. Actually the PSDs generated by the tool mentioned above do not include all the error sources, but only account for the high-order part of the global error budget. The error sources not included in the calculation are related to the NGS WFS errors and to the implementation errors. These additional errors have therefore been included as corrective factor in the Strehl Ratio estimates. The global error budget, including all error sources, amounts to approximately $300 \mathrm{~nm}$ RMS, averaged over a 2 arcmin FoV. The overall performance, for a seeing FWHM $=0.8^{\prime \prime}$ at $\lambda=0.5 \mu \mathrm{m}$, is shown in figure 3 and in table 1.

The sky coverage has been estimated by means of Monte Carlo simulations of random asterisms, with star densities derived from the TRILEGAL model (Girardi et al. [12]). The calculations are based 


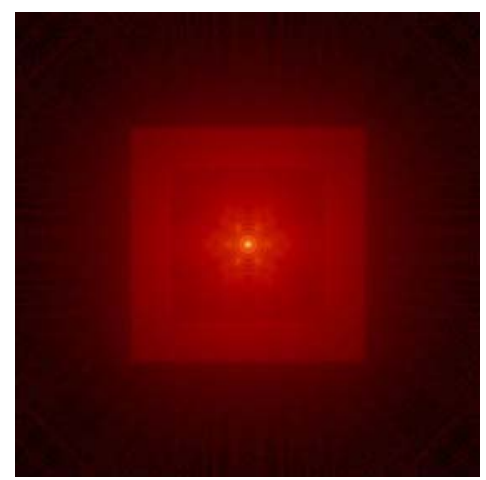

Fig. 2. Sample PSF at the central wavelength of the $\mathrm{K}_{\mathrm{s}}$ band $(\lambda=2.16 \mu \mathrm{m})$. The display size corresponds to approximately $2.7^{\prime \prime} \times 2.7^{\prime \prime}$ on sky. The square and hexagonal patterns in the PSF halo are related to the fitting and aliasing errors; the wide halo is related to the uncorrected turbulence.

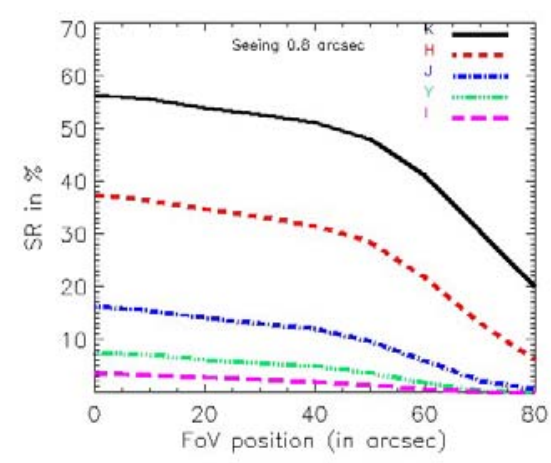

Fig. 3. Plot of Strehl Ratio vs. radial distance from the field center.

Table 1. Strehl Ratio for different wavelengths averaged over different FoV diameters.

\begin{tabular}{rrrrrr}
\hline FoV & $\mathrm{K}_{\mathrm{s}}(\lambda=2.16 \mu \mathrm{m})$ & $\mathrm{H}(\lambda=1.65 \mu \mathrm{m})$ & $\mathrm{J}(\lambda=1.215 \mu \mathrm{m})$ & $\mathrm{Y}(\lambda=1.021 \mu \mathrm{m})$ & $\mathrm{I}(\lambda=0.9 \mu \mathrm{m})$ \\
\hline $120^{\prime \prime}$ & 0.48 & 0.29 & 0.10 & 0.04 & 0.02 \\
$60^{\prime \prime}$ & 0.54 & 0.34 & 0.14 & 0.06 & 0.03 \\
$20^{\prime \prime}$ & 0.56 & 0.37 & 0.16 & 0.07 & 0.03 \\
$10^{\prime \prime}$ & 0.56 & 0.37 & 0.16 & 0.08 & 0.04 \\
\hline
\end{tabular}

on the NGS WFS design described in section 2. The sky coverage estimate at the North Galactic Pole is presented in table 2: it is expressed by the probability of occurrence of a given Strehl Ratio averaged over a FoV $=60^{\prime \prime}$.

The MCAO module is required to enable very accurate relative photometry and astrometry. The relative photometric accuracy is expected to be in the order of 0.03 magnitude RMS; the relative astrometric accuracy required by the imaging camera MICADO is in the order of 0.05-0.1 mas. Concerning the relative photometry requirements, preliminary studies have been carried out on synthetic images simulated using the PSFs described above. The main conclusion is that the MCAO performance is able to ensure the requested photometric accuracy. To assist the data analysis a study has been started to develop a specific PSF model, including the field variation. The astrometric accuracy has been evaluated on simulated images, investigating the effect of the PSF shape and variation across the field and the impact of the seeing variations. As far as these effects are concerned, the MCAO performance is 
Table 2. Expected Strehl Ratio performance vs. sky coverage at the Galactic Pole. The Strehl Ratio reported here is averaged over a FoV $=60^{\prime \prime}$. The NGS WFS probes are assumed to be positioned in order not to obscure this FoV.

\begin{tabular}{rrrrrr}
\hline $\mathrm{K}_{\mathrm{s}}(\lambda=2.16 \mu \mathrm{m})$ & $\mathrm{H}(\lambda=1.65 \mu \mathrm{m})$ & $\mathrm{J}(\lambda=1.215 \mu \mathrm{m})$ & $\mathrm{Y}(\lambda=1.021 \mu \mathrm{m})$ & $\mathrm{I}(\lambda=0.9 \mu \mathrm{m})$ & Sky coverage \\
\hline 0.54 & 0.34 & 0.14 & 0.06 & 0.03 & $39 \%$ \\
0.52 & 0.32 & 0.13 & 0.05 & 0.03 & $50 \%$ \\
0.42 & 0.22 & 0.06 & 0.02 & $<0.01$ & $80 \%$ \\
\hline
\end{tabular}

able to ensure the requested accuracy. A more detailed analysis of the astrometric errors, including atmospheric effects, shall have to be pursued further in the future, using PSFs obtained by end-to-end simulations. A clear aspect is that the achievement of such a high astrometric accuracy will require an accurate calibration of the distortions due to the optics and to the MCAO technique itself.

To draw few conclusions about the currently estimated performance, the MAORY module provides a corrected field of 2 arcmin diameter (up to 2.6 arcmin diameter, if one considers the whole field available for NGS search) corrected with good quality (average $\mathrm{SR} \sim 0.5$ in $\mathrm{K}_{\mathrm{s}}$ over 2 arcmin), with an exceptional correction uniformity (RMS variation of SR lower than 0.05 over the full FoV) and with high sky coverage. The sky coverage is obtained in a robust way, because it is based on a high level of closed loop correction over the whole NGS search FoV. These aspects constitute the key features of this MCAO module.

This work was supported by the European Community (Framework Programme 6, ELT Design Study, contract No 011863; Framework Programme 7, Preparing for the Construction of the European Extremely Large Telescope, contract No INFRA-2007-2.2.1.28) and by the European Southern Observatory

(Agreement No 16669/ESO/INS/07/17243/LCO).

\section{References}

1. Gilmozzi R., Spyromilio J., Proc. SPIE 7012, (2008) page 701219-1

2. Hubin N., Review of AO systems studied for the E-ELT, this Symposium.

3. Rigaut F. et al., Calibration schemes in MCAO, this Symposium.

4. Ellerbroek B., Adaptive Optics Systems for the Thirty Meter Telescope, this Symposium.

5. Herriot G. et al., NFIRAOS - first light adaptive optics system for TMT, this Symposium.

6. Davies R. et al., AO Requirements of MICADO, the E-ELT imaging camera, this Symposium.

7. Foppiani I. et al., MCAO for the E-ELT: preliminary design overview of the MAORY module, this Symposium.

8. Conan J.-M. et al., Wide Field AO design rules for ELTs, this Symposium.

9. Ellerbroek B., Rigaut F., J. Opt. Soc. Am. A Vol. 18, No. 10, (2001), page 2539-2547

10. Pfrommer T. et al., High-resolution results from the UBC-lidar experiment for the Thirty Meter Telescope, this Symposium.

11. Petit C. et al., Wide Field AO simulation for ELT: fourier and global E2E approaches, this Symposium.

12. Girardi L., Groenewegen M.A.T., Hatziminaoglou E., da Costa L., Astron. \& Astroph. 463, (2005) page 895 . 